\section{Evaluation of Gold Nanoparticle Size Effect on Dose Enhancement Factor in Megavoltage Beam Radiotherapy Using MAGICA Polymer Gel Dosimeter}

\author{
Behrouzkia Zh. ${ }^{1}$, Zohdiaghdam R. ${ }^{2 *}$, Khalkhali H. R. ${ }^{3}$, \\ Mousavi F. ${ }^{4}$
}

\begin{abstract}
Background: Gold nanoparticles (GNPs) are among the most promising radiosensitive materials in radiotherapy. Studying the effective sensitizing factors such as nanoparticle size, concentration, surface features, radiation energy and cell type can help to optimize the effect and possible clinical application of GNPs in radiation therapy. In this study, the radiation sensitive polymer gel was used to investigate the dosimetric effect of GNP size in megavoltage (MV) photon beam radiotherapy.
\end{abstract}

Material and Methods: GNPs with the size of $30 \mathrm{~nm}, 50 \mathrm{~nm}$ and $100 \mathrm{~nm}$ in diameter were used. Transmission electron microscope (TEM) and dynamic light scattering (DLS) were applied to analyze the size of nanoparticles. The MAGICA polymer gel was synthesized and impregnated with different sizes of GNPs. The samples were irradiated with $6 \mathrm{MV}$ photon beam and 24 hours after irradiation, they were read using a Magnetic Resonance Imaging (MRI) scanner. Macroscopic Dose Enhancement Factor (DEF) was measured to compare the effect of GNP size. The MAGICA response of the 6MV x-ray beam was verified comparing Percentage Depth Dose (PDD) curve extracted from polymer gel dosimetry and Treatment Planning System (TPS).

Results: MAGICA polymer gel dose response curve was linear in the range of 0 to $10 \mathrm{~Gy}$. DEFs by adding $30 \mathrm{~nm}, 50 \mathrm{~nm}$ and $100 \mathrm{~nm}$ GNPs were 1.1, 1.17 and 1.12 , respectively. PDD curves of polymer gel dosimeter and treatment planning system were in good agreement.

Conclusion: The results indicated a substantial increase in DEF uses a MV photon beam in combination with GNPs of different sizes and it was inconsistent with previous radiobiological studies. The maximum DEF was achieved for 50nm GNPs in comparison with $30 \mathrm{~nm}$ and $100 \mathrm{~nm}$ leading to the assumption of self-absorption effect by larger diameters. According to the outcomes of this work, MAGICA polymer gel can be recommended as a reliable dosimeter to investigate the dosimetric effect of GNP size and also a useful method to validate the current radiobiological and simulation studies.

\section{Keywords}

Gold Nanoparticle, Polymer Gel, Dosimetry, Radiotherapy

\section{Introduction}

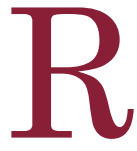
adiotherapy is an important method for the treatment of cancer and nearly $50 \%$ of cancer patients receive radiotherapy during their treatment [1]. However, the presence of resistant cells to radiation is the cause of most failure and tumor relapse. Radiation sensitizers are agents that enhance the sensitivity of tumor cells to radiation
${ }^{1} \mathrm{PhD}$ of Medical Physics, Urmia University of Medical Science, School of Medicine, Urmia, Iran

${ }^{2} \mathrm{PhD}$ of Medical Physics, Urmia University of Medical Science, School of Para Medicine, Urmia, Iran

${ }^{3}$ PhDs of Biostatics,

Patient Safety Research Center, Urmia University of Medical Sciences,

Urmia, Iran

${ }^{4} \mathrm{MSc}$ in Medical Physics, Urmia University of Medical Science, School of Medicine, Urmia, Iran

*Corresponding author:

R. Zohdiaghdam

PhD of Medical Phys-

ics, Urmia University of

Medical Science, School

of Para Medicine, Urmia, Iran

E-mail: zohdiaghdam@ gmail.com

Received: 18 September 2018 Accepted: 7 January 2019 
damages. Recently, there has been a considerable increase in studying high $\mathrm{Z}$ (atomic number) nanomaterials mainly gold $(Z=79)$, as a radiosensitizer [2].The unique properties of gold have caused it to be suggested as the best candidate in nanoparticle radiotherapy. Radiobiological studies, including in-vivo and invitro researches, along with $\mathrm{MC}$ simulations have focused on investigating the effective sensitizing factors such as the nanoparticle size, concentration, surface features, radiation energy and cell type which can help to optimize the effect and possible clinical application of GNPs in radiation therapy [2-5]. Potential dose enhancement in kilovoltage $\mathrm{KV}$ ) radiation, is well-known and has been confirmed by many studies. However, since clinically, MV radiation is used for the most of the radiotherapy practices rather than $\mathrm{KV}$ energies, a much wider clinical application could be achieved if GNP radiosensitization was obtained using MV sources [6-7]. While the majority of studies have reported low or no increases in MV energies, there are some differences between biological and physical achievements using high energy clinical beams [6], which make it necessary to improve the measurement methods for studying GNP application in radiotherapy. Another considerable factor is GNP size that may play an important role in both radiation toxicity and sensitization [8-9]. While most researches have been performed investigating the smaller (less than 2nm) GNPs, 50nm GNPs have shown a better radiosensitization effect [10]. However, size has been reported to be a less relevant factor in sensitization by some studies verifying both large (up to 100nm) and small (2nm) diameters [11-12]. The differences between studies outcomes introduce some more investigations to help the current results coming into a close conclusion. Recently, using a polymer gel dosimeter impregnated with GNP has been suggested as a reliable method to perform the dosimetric investigation of GNP application in radiotherapy. Previous studies considering beam energy and GNP concentration utilizing various polymer gels have been accomplished successfully [13-14]. Therefore, the dosimetric study of GNP using a tissue equivalent polymer gel would be a remarkable method to verify the current biological and simulation studies and also help to justify the applicability of GNP in clinical environments. This study uses MAGICA (Methacrylic Ascorbic in Gelatin Initiated by Copper with Agarose added) polymer gel as a radiation dosimeter to evaluate the effect of GNPs with large diameters (30, 50 and $100 \mathrm{~nm})$ on the macroscopic dose enhancement factor in MV radiation therapy.

\section{Material and Methods}

\section{A. Gold nanoparticle}

GNPs of $30 \mathrm{~nm}, 50 \mathrm{~nm}$ and $100 \mathrm{~nm}$ size were purchased from PNF Co. (Tehran, Iran) in the form of aqueous solution with $0.5 \mathrm{mg} / \mathrm{ml}$ (500ppm) concentration. Nanoparticles size was determined using DLS (Malvern, UK) and TEM (Philips, 30M-10, Netherlands). Figure 1 shows the TEM images of GNPs.

\section{B. Gel fabrication}

The MAGICA polymer gel used in this study was prepared using Fong et al. [15] method with a little different due to adding agarose. First, the water was divided into five flasks to dissolve each material. About $60 \%$ of the total HPLC de-ionized water was used in gelatin and left in the room temperature to swell for about one hour. Two electrical heater-stirrer units were used to heat and solve the solutions. Gelatin was heated up to $50^{\circ} \mathrm{C}$ until a clear solution was obtained. About $30 \%$ of water which had been heated up to $70^{\circ} \mathrm{C}$ was used to dissolve the agarose, then heating and stirring continued up to about $90^{\circ} \mathrm{C}$ until all the agarose were solved. Then both gelatin and agarose solution were allowed to cool until about $47^{\circ} \mathrm{C}$, at this time agarose solution was added to gelatin and stirring continued. Stirring never 


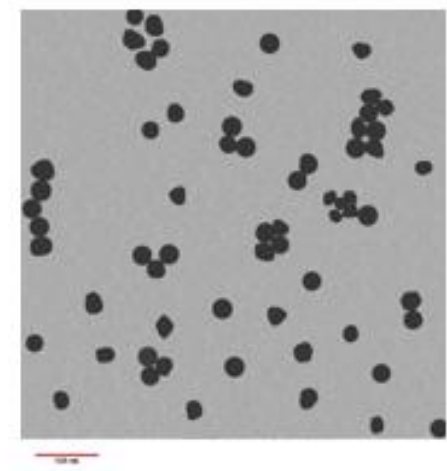

a

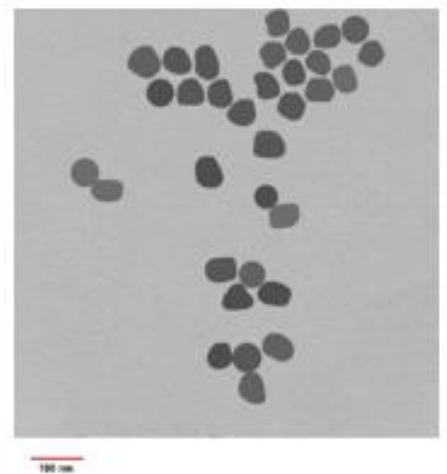

b

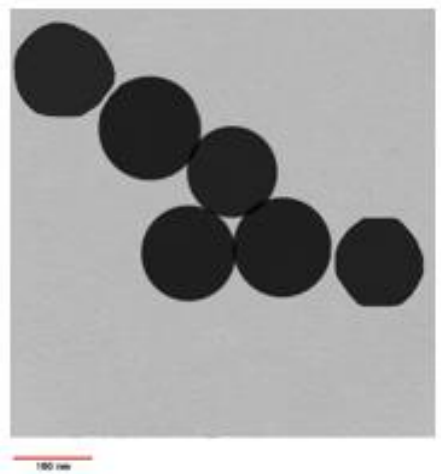

c

Figure 1: TEM images of GNPs a. $30 \mathrm{~nm}$ GNP b. $50 \mathrm{~nm}$ GNP and c. $100 \mathrm{~nm}$ GNP.

stopped before the end of fabrication. At $45^{\circ} \mathrm{C}$ hydroquinone, which had been solved in about $5 \%$ of water, was added to the mixture. The remaining $5 \%$ of water was divided into two portions for dissolving ascorbic acid and copper (II) sulphate. They were added to the mixture when the temperature was about $37^{\circ} \mathrm{C}$. Methacrylic acid was added at the end [16]. When the preparation of MAGICA was completed, the solution separated into four portions, one part for MAGICA and three parts for incorporating GNPs with different sizes. According to previous works that achieved a maximum dose enhancement for this concentration, a constant concentration of $0.1 \mathrm{mM}$ was used in this study [13]. After preparation of MAGICA and Gold Nano-MAGICA (GN-MAGICA), they were poured in calibration tubes for being kept in the refrigerator at about $4^{\circ} \mathrm{C}$.

\section{Irradiation procedure}

Gel samples were irradiated with 6MV photon beam from a medical linear accelerator (Siemens, Germany). The calibration tubes were set up in $50 \times 50 \times 50 \mathrm{~cm}^{3}$ water phantom at the Source to Surface Distance $(\mathrm{SSD})=100$ $\mathrm{cm}$ and field size of $30 \times 30 \mathrm{~cm}^{2}$ to cover all calibration tubes. They were exposed to radiation with specific doses in the range of $0-10$ Gy $(0,2,5,7,10 \mathrm{~Gy})$. For verification of the MAGICA response of the $6 \mathrm{MV}$ x-ray beam, an appropriate container of sizes $10 \times 10 \times 15 \mathrm{~cm}^{3}$ was made from plexiglass and irradiated with a field size of $10 \times 10 \mathrm{~cm}^{2}$ and SSD of $100 \mathrm{~cm}$ to obtain the PDD in the gel and then compare it with the TPS curve for the same irradiation condition, a single dose of 7Gy was applied.

\section{MRI reading and processing}

MRI was used to read out the polymer gel dosimeter. To ensure that the polymerization mechanism has completely done, 24 hours after irradiation all gel samples were scanned by a $1.5 \mathrm{~T}$ Philips (Achieva, Netherlands) MRI scanner using a head coil. A multi-spin echo sequence with 32 echoes, echo time of $15 \mathrm{~ms}$, repetition time of $3000 \mathrm{~ms}$, slice thickness $3 \mathrm{~mm}$ and the field of view $300 \mathrm{~mm}$ were used. Prior to the imaging process, samples were kept in the MRI room for about 4 hours to minimize the possible temperature gradient during imaging. $\mathrm{R}_{2}$ maps were extracted from MR images using MATLAB software (version 8.4).

\section{Results}

MAGICA dose-response curve is shown in Figure 2 and represents a linear relationship between $R_{2}$ values and dose in the range of 0-10 Gy (with a good approximation of $\mathrm{R}^{2}=$ 0.9812 ). Gel response of the $6 \mathrm{MV}$ X-ray beam was characterized by comparing the experi- 
mental and TPS data of PDD curve which is shown in Figure 3. The Dose Difference (DD) was less than $4 \%$ for all points representing a good agreement between experimental and calculated data. DD curve is also presented in Figure 4.

The chemical interaction between gel and GNP must be take into account since it can interfere the dose measurement [17]. According to this the signals of non-irradiated tubes containing and not containing GNPs were evaluated and shown in Figure 5. Chemical interaction between GNPs with different sizes and MAGICA polymer gel could be ignored

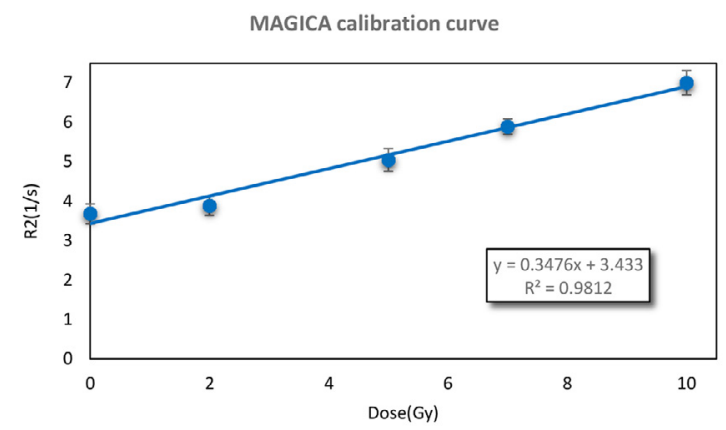

Figure 2: MAGICA calibration curve, presents a linear relation between $R_{2}$ and Dose $\left(R^{2}=0.995\right)$.

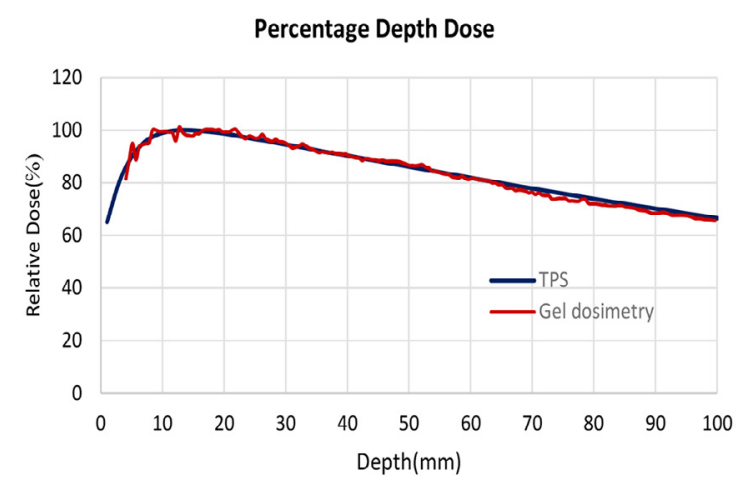

Figure 3: Comparison of PDD curves with gel dosimetry and TPS, DD for all points was less than $4 \%$ which shows a good agreement between experimental and TPS data.

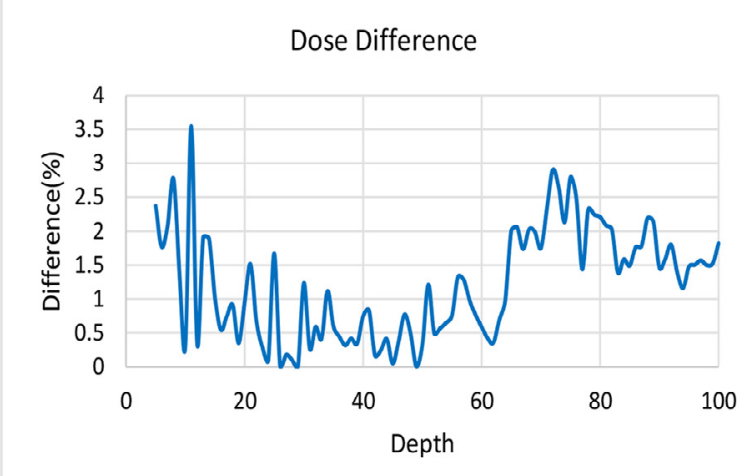

Figure 4: Dose Difference between MAGICA polymer gel dosimeter and Treatment Planning System (DD less than 4\% for all points).

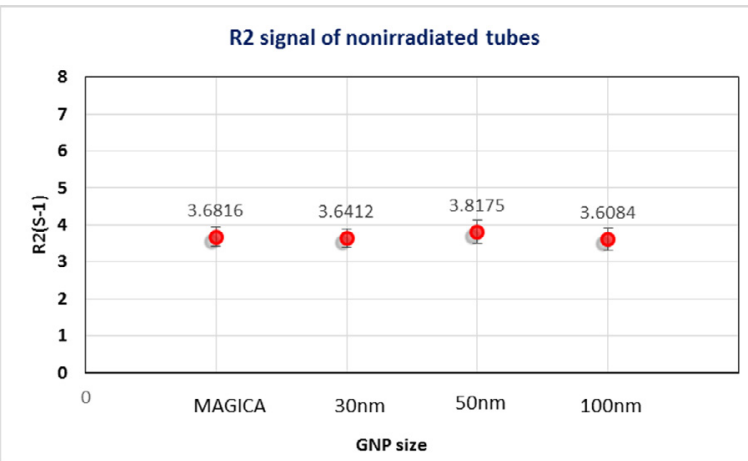

Figure 5: $R_{2}$ signal of non-irradiated tubes containing and not containing GNPs, $R_{2}$ signal variation less than $0.5 \%$.

because of low $\mathrm{R}_{2}$ signal variation (less than $0.5 \%$ ).

The dose response curves for all the three GN-MAGICA samples containing $30 \mathrm{~nm}$, $50 \mathrm{~nm}$ and $100 \mathrm{~nm}$ GNPs were calculated as it was achieved for the pure MAGICA and the ratio of the slopes (known as the sensitivity) was taken as the dose Enhancement Factor (DEF). Figure $6 \mathrm{a}, \mathrm{b}$ and $\mathrm{c}$ are presenting the dose response curves. It can be seen that in the presence of GNPs with different sizes the linear relation between $R_{2}$ values and dose in the range of 0 to 10 Gy dose did not change.

According to Figure $6 \mathrm{a}, \mathrm{b}$ and $\mathrm{c}$ the increase in gel sensitivity due to the presence of GNPs with different sizes can be seen. Table 1 also 

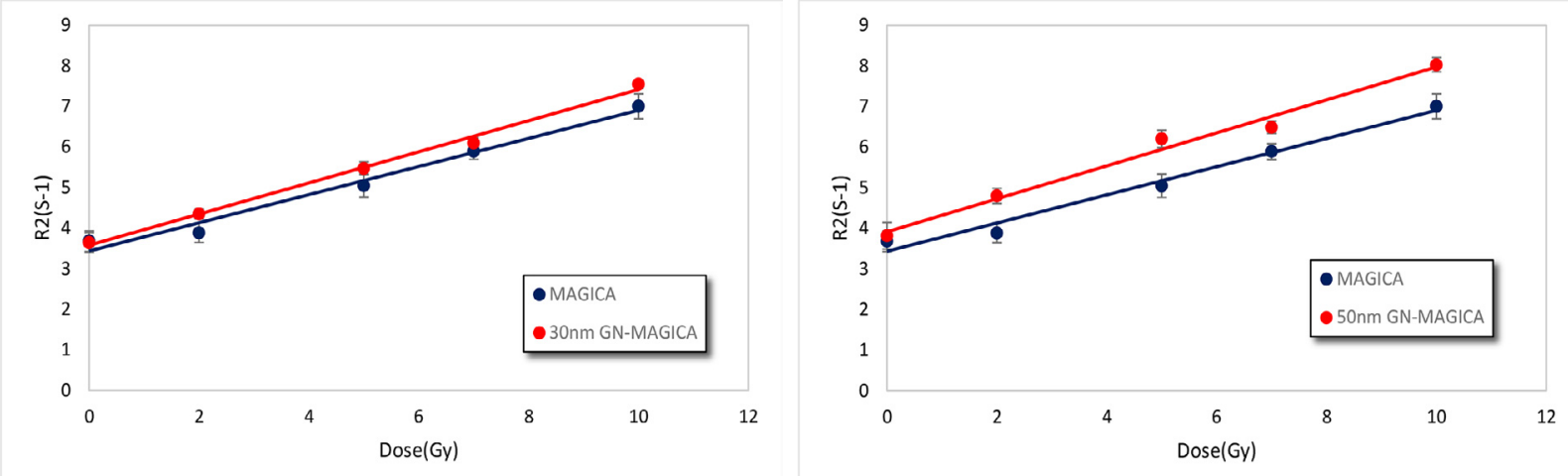

A

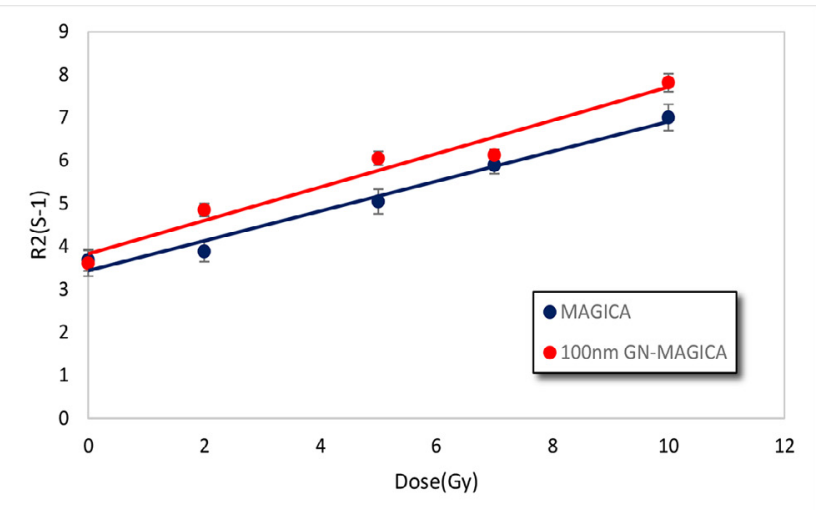

C

Figure 6: The dose response curves of GN-MAGICA samples with different sizes of GNP. (a) Dose response curve for 30nm GN-MAGICA, (b) dose response curve for 50nm GN-MAGICA and (c) dose response curve for $100 \mathrm{~nm}$ GN-MAGICA. All curves show a linear relation in the range of 0 to $10 \mathrm{~Gy}$.

summaries the DEF and sensitivity of gel samples.

Achieved macroscopic DEFs for $30 \mathrm{~nm}$, $50 \mathrm{~nm}$ and 100nm GNPs according to Table 1, are $1.1,1.17$ and 1.12 , respectively. The $50 \mathrm{~nm}$ GNP gain the maximum DEF in comparison with 30 and $100 \mathrm{~nm}$ GNP.

\section{Discussion}

The macroscopic DEFs due to the presence of $30 \mathrm{~nm}, 50 \mathrm{~nm}$ and $100 \mathrm{~nm}$ GNPs using clinical $6 \mathrm{MV}$ photon beam were evaluated in this study and the DEFsof 1.1, 1.17 and 1.12 for 30, 50 and $100 \mathrm{~nm}$ GNPs were achieved, respectively. The DEFs in this study, which are presented in Table 1, were derived from
Table 1: Representing the DEF achieved due to the presence of $30 \mathrm{~nm}, 50 \mathrm{~nm}$ and $100 \mathrm{~nm}$ GNPs.

\begin{tabular}{ccc} 
Gel Type & $\begin{array}{c}\text { Sensitivity } \\
\text { R-squared } \\
\text { value }\end{array}$ & DEF \\
\hline MAGICA & $\begin{array}{c}0.3476 \mathrm{~S}^{-1} \mathrm{~Gy}^{-1} \\
\mathrm{R}^{2}=0.9812\end{array}$ & \\
\hline 30nm GN-MAGICA & $\begin{array}{c}0.3838 \mathrm{~S}^{-1} \mathrm{~Gy}^{-1} \\
\mathrm{R}^{2}=0.9944\end{array}$ & 1.1 \\
\hline 50nm GN-MAGICA & $\begin{array}{c}0.4062 \mathrm{~S}^{-1} \mathrm{~Gy}^{-1} \\
\mathrm{R}^{2}=0.9852\end{array}$ & 1.17 \\
\hline 100nm GN-MAGICA & $\begin{array}{c}0.3889 \mathrm{~S}^{-1} \mathrm{G} y^{-1} \\
\mathrm{R}^{2}=0.962\end{array}$ & 1.12
\end{tabular}


the ratio of the dose-response curve sensitivity of the GN-MAGICA and pure MAGICA polymer gel. The calibration curves of GNMAGICA containing 30, 50 and 100nm GNPs in comparison with MAGICA are displayed in Figure $6 \mathrm{~A}$ to $\mathrm{C}$, respectively. The mechanism underlying the increase in slope of dose-response curve is simply defined by the amount of polymerization occurring in the polymer gel. Under irradiation, radical species which are produced from the radiolysis of water initiate the polymerization in gel dosimeter. The extent of radiation-induced polymerization is a dose dependent and hence, it is expected that for a given dose, the gel dosimeter loaded with a radio sensitizer, undergoes a larger amount of polymerization due to the more radical generation. The degree of polymerization is then related to the spin-spin relaxation rate $\left(\mathrm{R}_{2}=1 / \mathrm{T} 2\right)$ measured by MRI [17-19]. Therefore, the $\mathrm{R}_{2}$-dose sensitivity will be higher for the polymer gel containing a radio sensitizer like GNPs. As it is presented in Table 1, the highest sensitivity develops for 50, 100 and $30 \mathrm{~nm}$ GNPs, respectively which results to a maximum DEF for 50nm GNP. There is a comparable in-vitro study by Chitrani et al. [10]. Who investigated the radio sensitization effect of 14, 50 and 74nm GNPs in both KV and MV energies? In this study, 50nm GNP showed the highest sensitization effect and an enhancement factor of 1.17 was reported for 6MV photon beam which is inconsistent with our achievement. The author's discuss the difference in radio sensitization according to the number of GNPs internalized per cell since it was higher for 50nm GNP in spite of the same extracellular gold concentration. In our study also, 50nm GNP resulted in a larger amount of polymerization which then lead to a higher DEF in comparison with 30 and $100 \mathrm{~nm}$ GNPs. Since the concentration was the same for all sizes, the most likely reason for small differences between DEF of 50 and $100 \mathrm{~nm}$ GNPs, could be due to the self-absorption effect by a larger diameter. However, a microscopic simulation study investigating 2, 50 and 100nm GNPs applying both KV and MV photon beams was carried out by Leung et al. [20], they came to the conclusion that self-absorption by particles can be ignored due to the larger portion of energy deposition outside the particles. It is also reported that GNPs with larger diameters lead to a greater dose enhancement due to the increase in total interaction number. Nevertheless, considering all components which were discussed in the article, including the effective range, deflection angle, radial dose distribution, energy and interaction processes of secondary electrons created during the interaction of photon beam with GNPs, the overall results may vary when multiple particles are present as in macroscopic studies, since in microscopic studies, just the manner of a single particle is investigated. In a macroscopic study by Mesbahi et al. and a nanodosimetric investigation by Lechman et al. considering the photon energy, nanoparticle size and concentration, size has shown to be a less relevant factor in dose enhancement and the number of photoelectric absorption for energies above the k-edge of gold atom [11-12]. They also have confirmed the effectiveness of GNP application in KV energies, while a negligible enhancement has been reported for MV energies. To discuss the differences between present and mentioned simulation studies, it is worth considering a recent study by McMahon et al [21] who calculated the nanodosimetric effect of a single GNP irradiated with MV energies. As a result of their investigation, a substantial dose in homogeneity was observed around the GNP that may contribute to the significant radio sensitization reported by some radiobiological studies in the range of MV energies. According to the cellular evaluation, it has been mentioned that GNPs tend to form large clusters up to hundreds of nanometers inside the vesicles. As GNPs get larger, their dose distribution properties become different from the smaller particles due to occurring a large portion of ionizing events within their 
matter. This might be close to the assumption of self-absorption resulting a lower DEF in the case of 100nm GNP in comparison with $50 \mathrm{~nm}$ particles in our study. On the other hand, in Leung et al. investigation, despite the more impressive application of GNPs in low energies, an interaction ratio of approximately 10 was achieved in higher energies that demonstrates an enhancement of secondary electron generation in the MV ranges [20]. This is in consistent with our study which presents a considerable dose enhancement for 6MV photon beam in combination with GNPs. Our result is also in close agreement with the study of Alqathami et al. [22] who used a novel multi compartment radiochoromic radiation dosimeter to measure the dose enhancement produced by $50 \mathrm{~nm}$ GNP irradiated with $100 \mathrm{KV}$ and 6MV x-ray beams. DEFs of 1.77 and 1.11 were obtained for KV and MV energies, respectively. A Close agreement between the outcomes of this article and previous studies would introduce the gel dosimeter as an appropriate method besides radiobiological and physical studies to find an accurate and optimal approach for the application of GNPs in radiotherapy

\section{Conclusion}

This article evaluated the macroscopic dose enhancement factor due to the presence of GNPs with different sizes in combination with $6 \mathrm{MV}$ clinical photon beam using MAGICA polymer gel dosimeter. The purpose of this study was to investigate the GNP size effect, mainly large sizes of 30, 50 and $100 \mathrm{~nm}$ in the range of $\mathrm{MV}$ energies where low or no increases have been predicted by physical studies. Comparing the $\mathrm{R}_{2}$-dose sensitivity of GN-MAGICA and pure MAGICA, the DEFs of $1.1,1.17$ and 1.12 were achieved for 30,50 and 100nm GNPs, respectively which shows for 50nm GNP, DEF was higher. It was assumed that the lower DEF of 100nm GNP in comparison with $50 \mathrm{~nm}$ particles could be due to the self-absorption effect by larger diam- eters. Our results were in a very close agreement with previous studies and this study introduces MAGICA polymer gel as a reliable dosimeter besides the radiobiological and simulation studies to achieve more accurate results for GNP application in radiotherapy.

\section{Acknowledgment}

The authors thank to the cellular and molecular Lab of Urmia University of Medical Science for using the facilities for gel fabrication and the authors also like to acknowledge the center of MRI imaging in Emam hospital of Urmia for cooperation in MRI imaging.

\section{Conflict of Interest}

None

\section{References}

1. Baskar R, Lee KA, Yeo R, Yeoh KW. Cancer and radiation therapy: current advances and future directions. Int J Med Sci. 2012;9:193-9. doi: 10.7150/ ijms.3635. PubMed PMID: 22408567; PubMed Central PMCID: PMC3298009.

2. Kwatra D, Venugopal $A$, Anant $S$. Nanoparticles in radiation therapy: a summary of various approaches to enhance radiosensitization in cancer. Translational Cancer Research. 2013;2:330-42.

3. Jain S, Coulter JA, Hounsell AR, Butterworth KT, McMahon SJ, Hyland WB, et al. Cell-specific radiosensitization by gold nanoparticles at megavoltage radiation energies. Int $J$ Radiat Oncol Biol Phys. 2011;79:531-9. doi: 10.1016/j.jirobp.2010.08.044. PubMed PMID: 21095075; PubMed Central PMCID: PMC3015172.

4. Jeremic B, Aguerri AR, Filipovic N. Radiosensitization by gold nanoparticles. Clinical and Translational Oncology. 2013;15:593-601.

5. Su XY, Liu PD, Wu H, Gu N. Enhancement of radiosensitization by metal-based nanoparticles in cancer radiation therapy. Cancer Biol Med. 2014;11:8691. doi: 10.7497/j.issn.2095-3941.2014.02.003. PubMed PMID: 25009750; PubMed Central PMCID: PMC4069802.

6. Butterworth KT, McMahon SJ, Currell FJ, Prise KM. Physical basis and biological mechanisms of gold nanoparticle radiosensitization. Nanoscale. 2012;4:4830-8. doi: 10.1039/c2nr31227a. PubMed PMID: 22767423.

7. Hainfeld JF, Dilmanian FA, Slatkin DN, Smilow- 
itz HM. Radiotherapy enhancement with gold nanoparticles. J Pharm Pharmacol. 2008;60:97785.

8. Zhang XD, Wu D, Shen X, Chen J, Sun YM, Liu $P X$, et al. Size-dependent radiosensitization of PEG-coated gold nanoparticles for cancer radiation therapy. Biomaterials. 2012;33:6408-19. doi: 10.1016/j.biomaterials.2012.05.047. PubMed PMID: 22681980.

9. Zhang XD, Wu D, Shen X, Liu PX, Yang N, Zhao $B$, et al. Size-dependent in vivo toxicity of PEGcoated gold nanoparticles. Int J Nanomedicine. 2011;6:2071-81. doi: 10.2147/IJN.S21657. PubMed PMID: 21976982; PubMed Central PMCID: PMC3181066.

10. Chithrani $D B$, Jelveh $S$, Jalali $F$, van Prooijen $M$, Allen C, Bristow RG, et al. Gold nanoparticles as radiation sensitizers in cancer therapy. Radiat Res. 2010;173:719-28. doi: 10.1667/RR1984.1. PubMed PMID: 20518651.

11. Lechtman E, Chattopadhyay N, Cai Z, Mashouf S, Reilly R, Pignol J. Implications on clinical scenario of gold nanoparticle radiosensitization in regards to photon energy, nanoparticle size, concentration and location. Phys Med Biol. 2011;56:4631-47.doi: 10.1088/0031-9155/56/15/001.

12. Mesbahi A, Jamali F, Garehaghaji N. Effect of photon beam energy, gold nanoparticle size and concentration on the dose enhancement in radiation therapy. Bioimpacts. 2013;3:29-35. doi: 10.5681/ bi.2013.002. PubMed PMID: 23678467; PubMed Central PMCID: PMC3648909.

13. Marques T, Schwarcke M, Garrido C, Zucolot V, Baffa 0, Nicolucci P, editors. Gel dosimetry analysis of gold nanoparticle application in kilovoltage radiation therapy. Journal of Physics: Conference Series; 2010. doi: 10.1088/1742-6596/250/1/012084.

14. Rahman WN, Wong CJ, Ackerly T, Yagi N, Geso M. Polymer gels impregnated with gold nanoparticles implemented for measurements of radiation dose enhancement in synchrotron and conventional radiotherapy type beams. Australas Phys Eng Sci
Med. 2012;35:301-9.doi: 10.1007/s13246-0120157-x.

15. Fong PM, Keil DC, Does MD, Gore JC. Polymer gels for magnetic resonance imaging of radiation dose distributions at normal room atmosphere. Phys Med Biol. 2001;46:3105-13.doi: 10.1088/00319155/46/12/303. PubMed PMID: 11768494.

16. Zahmatkesh M, Kousari R, Akhlaghpour S, Bagheri $\mathrm{S}$. MRI gel dosimetry with methacrylic acid, ascorbic acid, hydroquinone and copper in agarose (MAGICA) gel. Preliminary Proceedings of DOSGEL. 2004;2004.

17. Meesat R, Jay-Gerin JP, Khalil A, Lepage M. Evaluation of the dose enhancement of iodinated compounds by polyacrylamide gel dosimetry. Phys Med Biol. 2009;54:5909-17. doi: 10.1088/00319155/54/19/016. PubMed PMID: 19759404.

18. Baldock C, De Deene Y, Doran S, Ibbott G, Jirasek A, Lepage M, et al. Polymer gel dosimetry. Phys Med Biol. 2010;55:R1.

19. Ibbott GS, editor Applications of gel dosimetry. Journal of Physics: conference series; 2004.

20. Leung MK, Chow JC, Chithrani BD, Lee MJ, Oms $B$, Jaffray DA. Irradiation of gold nanoparticles by $x$-rays: Monte Carlo simulation of dose enhancements and the spatial properties of the secondary electrons production. Med Phys. 2011;38:62431. doi: 10.1118/1.3539623. PubMed PMID: 21452700.

21. McMahon SJ, Hyland WB, Muir MF, Coulter JA, Jain S, Butterworth KT, et al. Nanodosimetric effects of gold nanoparticles in megavoltage radiation therapy. Radiother Oncol. 2011;100:412-6. doi: $\quad 10.1016 /$ j.radonc.2011.08.026. PubMed PMID: 21924786.

22. Alqathami $M$, Blencowe $A$, Yeo UJ, Doran SJ, Qiao G, Geso M. Novel multicompartment 3-dimensional radiochromic radiation dosimeters for nanoparticle-enhanced radiation therapy dosimetry. International Journal of Radiation Oncology* Biology* Physics. 2012;84:e549-e55.doi: 10.1016/j.ijrobp.2012.05.029. 